\section{Out of the blue}

Scott Tremaine

Catastrophes and Evolution: 1stronomical Foundations. Edited by S. V. M. Clube. Cambridge University Press: 1989. Pp.239. £25, \$44.50.

GEOLOGISTS and astronomers attempt to deduce the origin and evolution of the Earth and the Universe from their present states. Difficulties arise because astronomical and geological timescales are so long that observations provide only a single snapshot of complex timedependent processes. To help interpret this snapshot, both disciplines rely on the paradigm of uniformitarianism, which asserts that natural laws are constant in space and time, and that past evolution should be explained through presently observable processes.

A weakness in this paradigm is that some important processes could occur rarely or last for brief periods, so that evolution proceeds mostly through a series of rare 'catastrophes' rather than at a steady rate. Two examples of proposed geological catastrophes, of very different degrees of plausibility, are the biblical deluge and the impact of a large extraterrestrial body at the Cretaceous/ Tertiary boundary. The theme of this book, a collection of lectures from a meeting of the British Association for the Advancement of Science held in 1988, is the conflict between uniformitarianism and catastrophism in geology, and the astronomical evidence that catastrophic events such as impacts by comets and meteors have been significant in geological and biological evolution.

A desirable goal for a book of this kind would be to provide an introduction for geologists to the relevant astronomical issues and vice versa. In this respect, there are several excellent articles here. Hallam provides a good historical survey of catastrophism in geology; Grieve's article is a short but authoritative review of terrestrial impact cratering; and Alvarez et al. give a brief, clear and provocative account of the current status of the hypothesis that the Cretaceous/Tertiary extinction resulted from a large impact. But the coverage of issues is uneven and somewhat parochial: one example is that there are no articles that make the case against the impact hypothesis for the Cretaceous/ Tertiary extinction; another is that the discussion of astronomical issues strongly reflects the views of the editor and his collaborators. The general reader would not know that Clube argues for is a radically unconventional world-view, in which comets are captured from starforming regions, giant comets with more than 100,000 times the mass of observed

comets populate the Solar System with dust and debris, redshifts may be gravitational rather than cosmological, and even general relativity and Friedmann models of the Universe are suspect.

To some extent, uneven coverage is inevitable in such a short book: the text

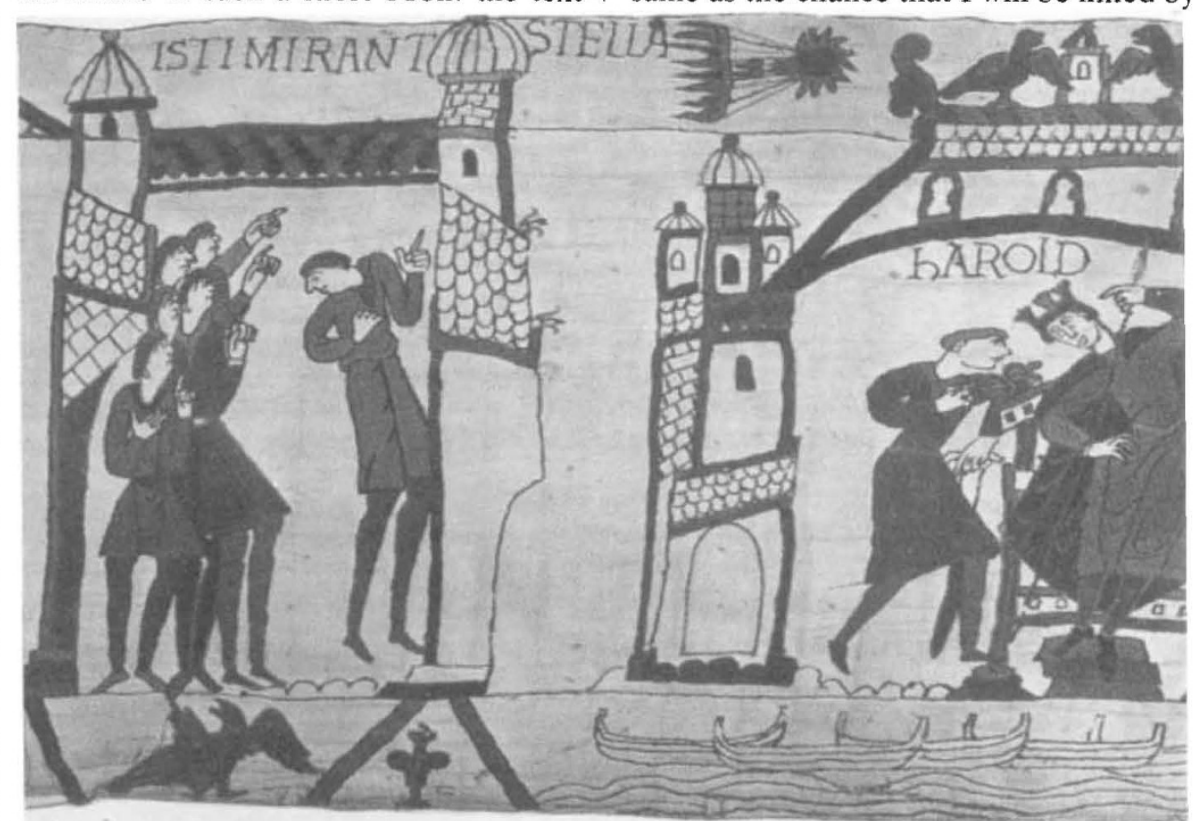

Halley's comet in 1065, 'a portent pregnant . covers only 240 pages, the page size is small and the typeface is large. Given this limitation, Catastrophes and Evolution provides a useful and readable but in-complete introduction to the role of astronomical catastrophes in geological and biological evolution.

None of the authors addresses one interesting aspect of catastrophism. If most organisms are killed once every 100 million years by the environmental consequences of a large impact, then each of us has roughly a one-in-a-million chance of dying from an unpredictable and unpreventable catastrophe of this kind. My local power company informs me that this is the same as the chance that I will be killed by

\section{Sound reading}

\section{David Young}

Arthropod Bioacoustics: Neurobiology and Behaviour. By Arthur W. Ewing. Edinburgh University Press: 1989. Pp.260. $£ 27.50$.

COMMUNICATION by sound in insects is now a thriving field of study, thanks mainly to the advent of convenient equipment for recording and analysing sound. Moreover, this is a form of behaviour that can be studied fruitfully at several different levels, from single nerve cells to selfish genes. I recently visited a laboratory where graduate students in neighbouring rooms were studying the neurobiology, ethology and physics of sound communication in a single group of insects.

Such combined studies have yielded some impressive results over the past couple of decades. In field and bush crickets (Orthoptera), for example, it has with dangers and disasters soon to come.

cancer caused by a major accident if I live within 8 kilometres of a nuclear reactor, or that I will be killed on my next airplane flight. In a certain sense, astronomical catastrophes provide a natural and immutable lower bound to the riskiness of human life.

Scott Tremaine is at the Canadian Institute for Theoretical Astrophysics, University of Toronto, Toronto M55 1A1, Canada. been possible to show which song features are important in natural behaviour and to find some of the neurons responsible for processing these features in the peripheral and central nervous systems. The neural and mechanical devices by which the specific song features are generated in the first place have also been elucidated. Being able to study acoustic behaviour at these different levels not only adds depth to the research but also makes it eminently suitable for inclusion in undergraduate courses on animal behaviour.

It is therefore good to see relevant aspects of the subject brought together in this book by Arthur Ewing, who is an experienced researcher on insect song. The word arthropod is used in the book's title, but in the nature of things the book is almost entirely about insects, except for the occasional interjection from shrimp or scorpion. Bioacoustics is interpreted broadly, as hinted at in the subtitle, with the range of topics covered moving from the physics of sound to the genetics and evolution of insect song. 